\title{
ANÁLISE DAS LACUNAS DA ABNT ISO 9001:2015 EM UMA EMPRESA DE TRANSPORTE DE CARGAS RODOVIÁRIAS FRACIONADAS
}

\author{
ANALYSIS OF ABNT ISO 9001:2015 GAPS IN A FRACTIONAL ROAD CARGO \\ TRANSPORT COMPANY
}

\author{
Livia Maria Delaci ${ }^{\mathrm{I}}$ \\ Glaucia Aparecida Prates ${ }^{\text {II }}$ \\ Elisangela Pereira Senno III \\ Rafael Altafin Galli ${ }^{\mathrm{IV}}$ \\ Lesley Carina do Lago Attadia Galli ${ }^{\mathrm{V}}$
}

\begin{abstract}
RESUMO
Este artigo visa analisar as lacunas da ISO 9001-2015 em uma empresa de transporte rodoviário propondo melhorias baseadas nos princípios da norma. Para o estudo de caso utilizou-se pesquisa bibliográfica, informações contidas no website da empresa, análise dos processos e acompanhamento da operação para analisar os gargalos nos procedimentos relacionados a norma ISO 9001-2015 que influenciam na qualidade do serviço prestado. É preciso que todos os colaboradores de uma organização estejam treinados e cientes dos critérios exigidos no sistema da qualidade geral da empresa e da ISO 9001-2015 norma essa apresentada no trabalho que demonstra a importância de ter funcionários capacitados e treinados em todas áreas da operação pois somente assim será possível a diminuição dos gargalos garantindo a total satisfação do cliente e aumentando a lucratividade da organização. Como resultados desse estudo de caso tendo como base as exigências da norma que define que todos os colaboradores devem ser treinados e avaliados os seus resultados de modo que o resultado dos trabalhos executados venham agregar valor para o cliente e para a organização é preciso existir organização ,comunicação e aumentar a carga horária de treinamentos, informar aos colaboradores a importância de se realizar suas obrigações da forma certa, priorizando a qualidade do serviço e das mercadorias que serão transportadas.
\end{abstract}

Palavras-chave: Transporte Rodoviário. ISO 9001:2015. Gestão da qualidade e Produtividade. Diferencial competitivo.

\footnotetext{
ABSTRACT

This study seeks to identify the quality and productivity factors required to guarantee the service with excellence in the highway modal. For the case study, bibliographic research was used, information contained in the company's website, analysis of the processes and monitoring of the operation to analyze the bottlenecks in the procedures related to ISO 9001-2015 that

I Aluno Especial no Mestrado em Administração da UNESP - FCAV- Jaboticabal - SP - Brasil. E-mail: liviadelaci@hotmail.com

II Profa. Dra. da UNESP - Itapeva - SP - Brasil. E-mail: g.prates@ unesp.br

III Mestrando no curso de Pós-graduação em Administração da UNESP - FCAV- Jaboticabal - SP - Brasil. Email: elis.senno@gmail.com

IV Prof. Ms. da Universidade Estadual de Minas Gerais. Passos-MG-Brasil. E-mail: rafaelaltafin@hotmail.com

v Profa. Dra. da UNESP - FCAV- Jaboticabal - SP - Brasil. E-mail: lesleyattadia@ @cav.unesp.br
} 


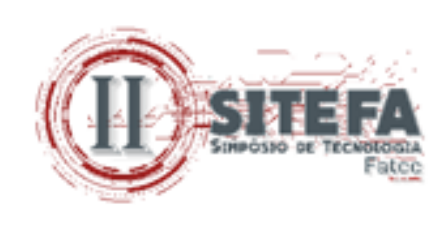

influence the quality of the service provided. It is necessary that all the employees of an organization are trained and aware of the criteria required in the general quality system of the company and ISO 9001-2015 standard presented in the work that demonstrates the importance of having trained and trained in all areas of the operation because only this way it will be possible to reduce the bottlenecks guaranteeing total customer satisfaction and increasing the profitability of the organization. However, it can be concluded that the quality management tools can be a competitive differential for the companies, since it allows the standardization of processes, reduction of costs, merchandise without damages and organization of work. As a result of this case study based on the requirements of the standard that states that all employees should be trained and evaluated their results so that the result of the work performed will add value to the customer and the organization, there must be organization, communication and increase training hours, inform employees of the importance of performing their duties in the right way, prioritizing the quality of service and the goods to be transported.

Keywords: Road transport. ISO 9001-2015. Quality management and Productivity. Differential competitive.

Data de submissão do artigo:11/07/2019.

Data de aprovação do artigo: 01/10/2019.

DOI:

\section{INTRODUÇÃO}

A avaliação da qualidade nos tempos atuais e os critérios exigidos pelos clientes na prestação de serviço estão cada vez mais exigentes. A prática da melhoria da qualidade da organização é fundamental para o sucesso da organização. A qualidade, no entanto, deve estar presente no processo como um todo e não apenas em partes do processo. O sistema de gestão da qualidade é importante para a organização pois conseguirá padronizar os processos, diminuir custos e gargalos que impedem que o serviço se torne eficiente.

A norma da ABNT ISO:9001:2015 bem como os seus requisitos exigem vários critérios a serem seguidos dentro da organização e por parte de todos os funcionários mencionando os itens relacionados aos processos e prestação de serviços que ao ser seguidos garantirá a qualidade e satisfação no mercado, por outro lado os impactos causados pelo não cumprimento dos procedimentos e forma de executar as etapas não estiverem de acordos com os princípios da norma é bem certo que a empresa não atingirá os seus objetivos e metas definidas como parâmetro de sucesso.

O sucesso de uma empresa depende de todos os setores, gestores e colaboradores motivados e que exerçam suas funções com organização e excelência. Para várias empresas a norma ABNT ISO 9001:2015 é usada como padrão de qualidade organizacional, pois permite a melhoria contínua em seus processos e serviços além de ganhar competitividade no mercado competitivo.

A norma ABNT ISO 9001:2015 é de fundamental importância para a empresa de transporte, pois garantem qualidade, profissionalismo no serviço prestado, as certificações comprovam que as empresas oferecem serviços qualificados garantindo agilidade e eficácia reduzindo custos e riscos durante o processo de transporte de cargas fracionadas.

Este trabalho tem como objetivo analisar as lacunas da norma da ABNT ISO 9001:2015 em uma empresa de transporte rodoviário de cargas fracionadas e propor melhorias relacionadas 


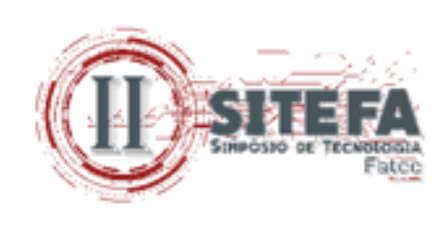

aos princípios exigidos na norma quando não são aplicados da forma correta podendo comprometer a qualidade do serviço prestado.

\section{PRINCÍPIOS DA QUALIDADE}

Os princípios de gestão da qualidade não se constituem em um conjunto de requisitos da qualidade. Entretanto, independentemente de seu tipo e porte, a organização precisa ter a capacidade de transpor a filosofia dos princípios para as práticas de seus processos (JASIULEWICZ-KACZMAREK, 2016). Os princípios de gestão da qualidade são bases conceituais que funcionam como premissas para o estabelecimento de sistemas de gestão da qualidade.

Buscando alinhar os princípios de gestão da qualidade às práticas organizacionais, Goharshenasan, Shahin e Esmaelian (2016, p.117-118) associam quatorze ferramentas de gestão da qualidade aplicáveis nos processos organizacionais aos sete princípios da qualidade estabelecidos na ISO 9000.

Basir e Davies (2016) em seu estudo sobre de caso um conselho municipal da Malásia, discutem que as organizações que adotam os princípios de gestão da qualidade necessitam desprender menos esforços com a manutenção de seus sistemas de gestão da qualidade.

Garza-Reyes, Rocha-Lona e Kumar (2014) em sua proposta de um modelo conceitual para implementação de sistemas de gestão da qualidade também comentam a importância da adoção dos princípios de gestão da qualidade para minimizar esforços e falhas nesse processo de implementação.

A seguir são elencados os documentos exigidos pela norma da ABNT ISO 9001:2015 Documentos e registros obrigatórios requeridos pela ISO 9001:2015

1. Escopo do SGQ (cláusula 4.3)

2. Política da qualidade (cláusula 5.2)

3. Objetivos da qualidade (cláusula 6.2)

4. Critérios para avaliação e seleção de fornecedores (cláusula 8.4.1)

5. Registros de monitoramento e medição de equipamento de calibração (cláusula

6. Registros de treinamento, habilidades, experiência e qualificações (cláusula 7.2)

7. Registros de análise crítica de requisitos de produto/serviço (cláusula 8.2.3.2)

8. Registro sobre análise crítica de saídas de projeto e desenvolvimento (cláusula

9. Registros sobre entradas de projeto e desenvolvimento (cláusula 8.3.3)

10. Registros de controles de projeto e desenvolvimento (cláusula 8.3.4)

11. Registros de saídas de projeto e desenvolvimento (cláusula 8.3.5)

12. Registros de mudanças em projeto e desenvolvimento (cláusula 8.3.6)

13. Características do produto a ser produzido e serviço a ser provido (cláusula

14. Registros sobre propriedade do cliente (cláusula 8.5.3)

15. Registros de controle de mudança de produção/provisão de serviço (cláusula

16. Registro de não conformidade de produto/serviço com critérios de aceitação (cláusula 8.6)

17. Registro de saídas não conformes (cláusula 8.7.2)

18. Resultados de monitoramento e medição (cláusula 9.1.1.) 


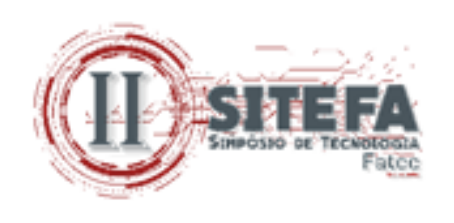

19. Programa de auditoria interna (cláusula 9.2)

20. Resultados de auditorias internas (cláusula 9.2)

21. Resultados de análises críticas da direção (cláusula 9.3)

22. Resultados de ações corretivas (cláusula 10.1)

\subsection{Transporte rodoviário de cargas fracionadas}

O transporte de cargas fracionadas é o ideal para atender a necessidade de embarcadores de pequenos e médios porte (OLIVEIRA,2007).

A carga fracionada é de pequena quantidade de mercadoria que sozinha não ocupa todo o equipamento que realizará o transporte. Esse tipo de transporte é definido pelo embarque de vários tipos diversificados de mercadorias organizadas no mesmo veículo de transporte (OLIVEIRA, 2007).

A empresa deste tipo realiza a coleta de qualquer quantidade e o tipo de carga compatível com o perfil em que atua no mercado.

É feito acordo comercial entre o transportador e o cliente onde são definidos preço, prazos de entregas e pagamentos.

Ainda para Oliveira (2007) As tecnologias de informação bem como uma boa gestão operacional são ferramentas consideradas estratégias para que a empresa de transporte se destaque no mercado.

Existem inúmeros KPIs- Key performance indicator ou indicadores que medem a qualidade e desempenho no transporte rodoviário, é analisado desde o departamento comercial onde é feito a negociação com o cliente até a entrega final para o cliente destinatário

Segundo Sinkova, Konecny e Liscak (2015) - não é et al. o sistema de Gestão da qualidade tem impacto positivo para rentabilidade da empresa, a qualidade do serviço é determinante para a demanda da empresa, os processos de infraestrutura, tecnologias de transporte, recursos humanos são quesitos fundamentais para a qualidade no serviço.

Para Martins et al (2011) como componente da logística, as expectativas, quanto ao desempenho do serviço de transportes, se tornaram mais complexas. A busca da eficiência dos processos logísticos fez com que outros atributos do serviço fossem incorporados ao bojo da tomada de decisão acerca dos transportes, como o cumprimento dos prazos, a transparência de custos e o desenvolvimento de serviços apropriados e integrados com fornecedores e clientes.

\section{METODOLOGIA}

Segundo Gil (2008) estudo de caso é definido pelo estudo de um ou de poucos objetos de modo a permitir o seu conhecimento amplo, detalhado e vem sendo utilizado com frequência por pesquisadores sociais porque a pesquisa serve para diferentes propósitos como: explorar situações da vida real quando não estão claramente definidos, descrever a situação do contexto de determinada situação, explicar variáveis causais de fenômeno com situação complexa que não permitem a utilização de levantamento e experimento.

O presente artigo foi realizado através de pesquisa bibliográficas relacionadas a norma da ABNT ISO 9001:2015 bem como seus conceitos aplicados no Transporte Rodoviário de cargas fracionadas e o estudo de caso.

Foi realizado um estudo de caso, em uma transportadora localizada na cidade de Ribeirão Preto- SP tendo como sua sede Matriz situada na cidade de Caxias do Sul -RS. Para a 


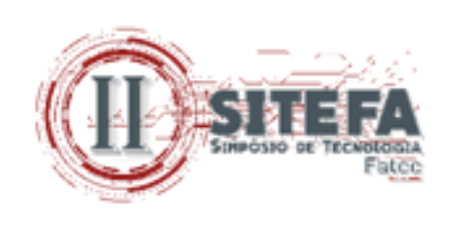

coleta dos dados foi realizado análises nos procedimentos exigidos da organização, acompanhamento dos processos desde o início da operação até a entrega final para o cliente.

Se fez necessário um estudo para compreender a Norma da ABNT ISO 9001:2015 e os seus Itens que relacionava ao tipo de falha na execução de cada processo. Após identificar os processos foram analisadas e registradas as falhas dos profissionais ao executar determinada função que impactava com alguns dos requisitos exigidos pela norma da ABNT ISO 9001:2015 fazendo com que os resultados de qualidade não fossem alcançados pela organização.

Foram realizadas análises dos processos e acompanhamento da operação a fim de analisar gargalos e falhas nos procedimentos contidos na Norma da ABNT ISO 9001-2015 que podem comprometer na qualidade dos produtos e no desempenho geral da empresa.

\section{RESULTADOS E DISCUSSÃO}

A empresa em estudo foi fundada em 1979 com objetivo de construir um negócio sólido para atender as necessidades dos seus clientes. No início começou a atender o mercado regional de transportes de cargas fracionadas com um serviço diferenciado, com metodologia, organização e segurança.

$\mathrm{O}$ estudo de caso apresentado neste trabalho é referente a unidade (filial) situada em Ribeirão Preto-SP que está atuando a 12 anos no mercado. Para implantação da norma a organização fundamentou-se no ciclo PDCA, fazendo todo planejamento, execução e avaliação dos processos para a definição dos objetivos a serem alcançados.

$\mathrm{Na}$ fase do planejamento reuniu-se a diretoria juntamente com os colabores envolvidos e foram definidas as estratégias usadas bem como ações, correções e melhorias no sistema. Em média o prazo para execução e finalização do trabalho foi de 2 anos, seguidos do cronograma de atividades e cada etapa do órgão certificador responsável pela auditoria final de certificação. A implantação do sistema de qualidade ocorreu dentro dos requisitos da norma ABNT ISO 9001 o que proporcionou a certificação dentro do prazo determinado. Com o desenvolvimento do comitê, os responsáveis faziam reuniões para que as informações do Sistema de qualidade envolvessem todos os departamentos. Com base nas exigências da Norma ABNT NBR ISO 90012015 criou-se a política de qualidade com foco na satisfação do cliente, melhoria contínua dos processos eficiência no Sistema de Gestão da Qualidade.

Por meio de todas as informações e descrição entres processos do Sistema de Gestão da qualidade criou-se o Manual de Gestão da Qualidade que era revisado atualizado, contendo todas as descrições dos processos. Todas as etapas foram executadas para atender as necessidades e exigências dos clientes, foram desenvolvidos treinamentos para os colaboradores a fim de serem capacitados para atender ao serviço do mercado de acordo com as exigências no sistema de Gestão qualidade. Em umas das fases do processo as auditorias internas se fizeram necessária para verificação da eficácia do Sistema de Qualidade.

Foram usadas ferramentas de qualidade para monitoramento e acompanhamento dos resultados como por exemplo PDCA e diagrama de Pareto. Por mais que tenham treinamentos de todos os envolvidos acredita-se que as ações e atitudes através de alguns colaboradores que não seguem os processos de acordo com a norma pode interferir nos melhores resultados e objetivos definidos pela organização

Quando não há colaboração de envolvimento de todos da organização pode impossibilitar a concretização dos objetivos definidos e o alcance das metas. 


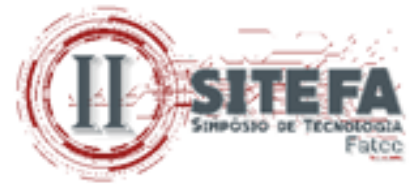

O quadro 1 a seguir demonstra as definições dos processos, falhas ao executá-los e os impactos relacionados aos itens da norma ABNT ISO 9001:2015 por não seguirem os procedimentos da forma correta.

Quadro 1 - Falhas nos processos identificados na empresa em estudo

\begin{tabular}{|c|c|c|}
\hline Processo & Função & Falhas no Processo \\
\hline Coleta & $\begin{array}{l}\text { Coletar mercadoria no cliente } \\
\text { mediante Nota } \begin{array}{l}\text { Fiscal e } \\
\text { volumes }\end{array} \\
\text { corretamente }\end{array}$ & $\begin{array}{l}\text { Falha na documentação, mercadoria não } \\
\text { embarca por falta de documento e falha } \\
\text { na informação, volumes embarcados } \\
\text { sem etiquetas }\end{array}$ \\
\hline $\begin{array}{l}\text { Recebimento de } \\
\text { cargas } \\
\text { transferência }\end{array}$ & $\begin{array}{l}\text { Descarregar mercadorias e } \\
\text { separar em rotas para entrega } \\
\text { ou transbordo }\end{array}$ & $\begin{array}{l}\text {-Manuseio de cargas incorreto- volume } \\
\text { avariado } \\
\text { - transferência de mercadoria para } \\
\text { destino errado- mal-uso da esteira } \\
\text { - rotas não organizadas, perdas de } \\
\text { volumes, demora no carregamento de } \\
\text { entrega } \\
\text { - falta de organização de mercadoria em } \\
\text { local adequado } \\
\text {-perda de documentação }\end{array}$ \\
\hline $\begin{array}{l}\text { Controle de carga } \\
\text { não conforme }\end{array}$ & $\begin{array}{l}\text { Organizar mercadorias não } \\
\text { conformes, volumes separados } \\
\text { e identificados. }\end{array}$ & $\begin{array}{l}\text {-Mercadoria em rota errada, perda de } \\
\text { volume, falta de informação em sistema }\end{array}$ \\
\hline $\begin{array}{l}\text { Distribuição de } \\
\text { cargas }\end{array}$ & $\begin{array}{l}\text { Realizar leitura de } \\
\text { carregamento de entrega e } \\
\text { separar paras as rotas de } \\
\text { entrega }\end{array}$ & $\begin{array}{l}\text { Não realiza leitura de carregamento com } \\
\text { coletor de dados ocorrendo troca de } \\
\text { mercadorias, falhas no carregamento, } \\
\text { falta de mercadoria }\end{array}$ \\
\hline $\begin{array}{l}\text { Mobile-app } \\
\text { celular- }\end{array}$ & $\begin{array}{l}\text { Monitoramento de motoristas } \\
\text { e entregas }\end{array}$ & $\begin{array}{l}\text { Internet ociosa, falta de bateria nos } \\
\text { aparelhos }\end{array}$ \\
\hline $\begin{array}{l}\text { Sistema } \\
\text { informação, redes } \\
\text { de internet }\end{array}$ & $\begin{array}{l}\text { Integração de processos } \\
\text { internos }\end{array}$ & Lento, trava, atrasos de embarque \\
\hline
\end{tabular}

Fonte: elaborado pelos autores (2019)

Com base nos estudos da Norma ABNT ISO 90012015 foram possíveis verificar que a organização não está seguindo de acordo com os itens exigidos. De acordo com o quadro 1 os processos analisados nesse estudo de caso demonstram falhas dos profissionais da empresa ao realizar os processos. 


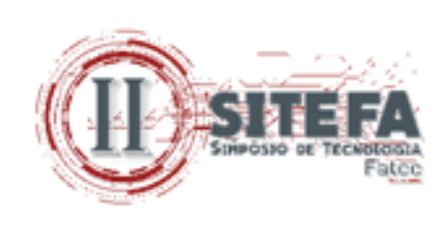

\section{CONCLUSÃO}

Este artigo teve como objetivo geral analisar as lacunas da ABNT ISO 9001:2015 em uma empresa de transporte rodoviário. Foi possível verificar os procedimentos executados pelos funcionários e a forma errada que realizavam determinadas etapas. A empresa possui sistema de gestão da qualidade e os processos são bem definidos para todas os departamentos da organização.

Ao realizar os estudos relacionados a norma e os requisitos exigidos para que a empresa esteja dentro das conformidades foi identificado falhas ao realizar os procedimentos, falta de comunicação entre a equipe e alguns recursos relacionados ao sistema de informação também se mostraram ineficientes para que a execução das atividades e a prestação do serviço em geral fosse concluída com êxito.

Como resultados desse estudo de caso tendo como base as exigências da norma que define que todos os colaboradores devem ser treinados e avaliados os seus resultados de modo que o resultado dos trabalhos executados venham agregar valor para o cliente e para a organização é preciso existir organização ,comunicação e aumentar a carga horária de treinamentos, informar aos colaboradores a importância de se realizar suas obrigações da forma certa, priorizando a qualidade do serviço e das mercadorias que serão transportadas.

Os funcionários devem estar cientes do não cumprimento dos requisitos da norma pode fazer com que a empresa tenha sérios prejuízos como: aumento de custos devido as avarias e extravios de mercadorias, funcionários desmotivados, ambiente de trabalho com gargalos gerando retrabalhos e somando todos esses fatores podem impactar na qualidade do serviço ofertado ocasionando a insatisfação dos clientes e impossibilitando o alcance dos objetivos da organização.

Conclui-se que somente quando todos os colaboradores da empresa executar suas atividades diárias com qualidade, comprometimento e eficiência garantindo que os requisitos exigidos pela norma ABNT ISO 9001:2015 estejam realmente sendo colocados em prática é o que de fato garantir que os produtos e serviços oferecidos estarão dentro do padrão de qualidade tornando a empresa competitiva e clientes cada vez mais satisfeitos.

\section{REFERÊNCIAS}

ASSOCIAÇÃO BRASILEIRA DE NORMAS TÉCNICAS (ABNT). ABNT NBR ISO 9001: sistemas de gestão da qualidade: requisitos. Rio de Janeiro, 2015.

BASIR, Siti Arni; DAVIES, John. ISO 9000 maintenance measures: the case of a Malaysian local authority. Total Quality Management \& Business Excellence, Abingdon, UK, 2016, p.2-17, abr

GARZA-REYES, Jose Arturo; ROCHA-LONA, Luis; KUMAR, Vikas. A conceptual framework for the implementation of quality management systems. Total Quality

Management \& Business Excellence, Abingdon, UK, v.26, n.12, p.1298-1310, jun. 2014. Disponível em: http://www.tandfonline.com/doi/abs/10.1080/14783363.2014.929254. Acesso em: 09 dez. 2017.

GIL, A. C. Métodos e técnicas de pesquisa social. São Paulo: Atlas, 2008 


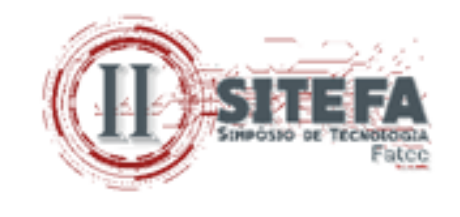

GOHARSHENASAN, A., SHAHIN, A., ESMAELIAN, M. Prioritisation of 14 quality management tools based on quality principles using integrated approach of multicriteria decision-making and importance-performance analysis - the case of Marjan Tile Company.

International Journal of Productivity and Quality Management, v. 18, n.1, 2016.

JASIULEWICZ-KACZMAREK, Małgorzata. ISO 9000:2015 quality management principles as the framework for a maintenance management system. Organizacja i Zarządzanie. Gliwice, n.69, p.49-65, 2016. Disponível em:

http://yadda.icm.edu.pl/yadda/element/bwmeta1.element.ekon-element-000171438900. Acesso em: 09 dez. 2017.

MARTINS R.S; XAVIER.W.S;FILHO.S.O.V; MARTINS.G.S, Transportation Management Oriented for Customers: Service Level Desired and Perceived, RAC, Curitiba, v. 15, n. 6, art. 7, pp. 1100-1119, Nov./Dez. 2011

OLIVEIRA.F. A, Transporte de carga fracionada: comportamento de compra dos lojistas de shopping centers em salvador. Salvador,2007.

SINKOVA.I; KONECNY.V; LISCAK.S, Measuring the quality impacts on the performance in transport company, Universidade de Nilina, Departamento de Estrada e Transportes Urbanos e Comunicações Univerzitná 8215/1, 01026 N̦ilina, República Eslovaca 2015,114 a 119 p. 